\title{
Five-wave mixing in molecular fluids
}

\author{
L C Dávila Romero, S R Meech and D L Andrews† \\ School of Chemical Sciences, University of East Anglia, Norwich NR4 7TJ, UK
}

Received 3 March 1997, in final form 2 September 1997

\begin{abstract}
The theory of a novel five-wave mixing process is developed for application to liquids and solutions. In its simplest implementation a sum-frequency signal is generated in a process which also accommodates features of optical phase conjugation. The flexibility in the beam geometry affords considerable scope for the study of the polarization and angular dependence. Together with the extensive possibilities for frequency tuning (and incidental exploitation of resonance features) the process proves to be powerfully dependent on molecular symmetry, and it lends itself to a very complete characterization of fourth-order optical nonlinearity.
\end{abstract}

\section{Introduction}

It is well known that optical three-wave mixing processes such as sum-frequency generation (SFG) are forbidden in fluid media except under certain rather specialized conditions. Principally, for coherent output, all three optical modes must have different frequencies and different polarization vectors; additionally, if the fluid is isotropic it must be comprised of chiral molecules (Rentzepis et al 1966, Andrews and Hands 1996). The former conditions on the radiation ensure that second harmonic generation (SHG) is forbidden, even through multipolar coupling, so that frequency doubling can be observable only through the operation of other exceptional mechanisms such as six-wave mixing (Andrews 1994, Allcock and Andrews 1997).

Against this background here we describe a novel five-wave mixing process which, though of a higher order than the familiar SHG and SFG, should offer conversion efficiencies broadly comparable to many well studied four-wave processes such as optical phase conjugation, with which it shares certain characteristics. The five-wave process offers considerable scope for the exploitation of resonances and (unlike SHG or SFG) it presents a flexible geometry - two features which greatly enhance its value for the fullest characterization of optical nonlinearity.

In what follows we first establish the logic that constrains the viability of five-wave mixing in a fluid, enabling a beam geometry that satisfies those constraints to be ascertained. The full theory is then developed through application of the methods of molecular quantum electrodynamics (QED), enabling the structure of the appropriate nonlinear susceptibility to be derived. Through irreducible tensor methods the rotational and distributional averaging necessary for proper representation of the material fluidity is then implemented to obtain directly applicable rate equations.

Particular attention is paid to the symmetry constraints governing the process. Molecular symmetry plays an important role in determining the basic condition (lack of inversion

$\dagger$ Corresponding author: email address: D.L.Andrews@uea.ac.uk 
symmetry) for the salient nonlinear optical susceptibility to be supported; however, the condition becomes more stringent when such species comprise the bulk of an isotropic fluid. Then it transpires that only chiral species can exhibit the five-wave interaction. Following a detailed analysis of the polarization behaviour of the process, we conclude with a discussion of special cases and potential applications.

\section{Initial considerations and constraints}

Since it has received relatively scant attention in the previously published literature, with a few key exceptions (Dubrovskii et al 1992, Shkurinov et al 1992, Koroteev 1995), it is appropriate first to review the general constraints and experimental viability of optical fivewave mixing. With five photons involved in each elementary interaction, the parametric processes of most interest are those: (i) involving the annihilation of three photons and the creation of two, or (ii) vice versa. The only other possibilities are (iii) the already well understood case of fourth harmonic generation (more generally, four-frequency addition), or (iv) the down-conversion counterparts. We focus on case (i) noting that the theory for (ii) is obtainable as the time inverse.

The simplest processes involving the annihilation of three photons and the creation of two are: (i) a trivial and essentially uninteresting higher-order contribution to SHG, representable in the conventional terminology as a process mediated by the nonlinear optical susceptibility $\chi^{(4)}(-2 \omega ;-\omega, \omega, \omega, \omega)$ and (ii) the $\frac{3}{2}$-frequency generation process invoking $\chi^{(4)}\left(-\frac{3}{2} \omega,-\frac{3}{2} \omega ; \omega, \omega, \omega\right)$. Both parametric interactions, and indeed any other such five-wave processes involving modes in collinear propagation, are forbidden in molecular fluids or other isotropic media for symmetry reasons. To discover an allowed process is then a matter of a progressive reduction in simplicity, by which is meant considering the involvement of an increasingly large number of optical modes, allowing for the possibility that two or more might have the same frequency.

Let us then consider processes that involve three photon frequencies, $\omega, \omega^{\prime}$ and $\omega^{\prime \prime}$, the first two designating input and the third, output. At the simplest level we can impose the SFG condition:

$$
\omega+\omega^{\prime}=\omega^{\prime \prime} .
$$

In order to satisfy energy conservation we must require that the other two photons involved in each elementary interaction, one annihilated and one created, have a common frequency, which again for simplicity we can take to be $\omega$. The created (non-signal) photon $\omega$ may be assumed to emerge through stimulated emission, i.e. following the direction of a throughput beam.

In summary, we can consider an elementary interaction involving the absorption of three photons $\left(\omega^{\prime}, \omega\right.$ and $\left.\omega\right)$ and the emission of another two $\left(\omega^{\prime \prime}\right.$ and $\left.\omega\right)$, thereby invoking a five-wave susceptibility expressible as $\chi^{(4)}\left(-\omega^{\prime \prime} ;-\omega, \omega, \omega, \omega^{\prime}\right)$. Viewed only with regard to the frequency arguments of the susceptibility, this could merely signify a higher-order contribution to SFG; however, as with degenerate four-wave phase conjugation mediated by $\chi^{(3)}(-\omega ;-\omega, \omega, \omega)$, novel features arise where modes having the same optical frequency impinge on the nonlinear medium from different directions.

In order to ascertain the constraints on directionality, the next step is to consider the requirement for wavevector matching. In general, this introduces a second condition, which in some cases can lead to trivial solutions such as $\omega^{\prime \prime}=\omega^{\prime}=\omega$ or $\omega^{\prime}=\omega$. To identify interesting but non-trivial solutions we can simplify the problem by considering the refractive index of the medium to be frequency independent-this is a simplification that we need 
not impose for the subsequent detailed calculations. With carefully chosen wavevector directions, phase matching can lead to the same conditions as the conservation of energy, thus affording a greater degree of experimental flexibility. The simplest processes are those involving just two (positive or negative) directions; we then conceive as the simplest nontrivial solution the beam geometry depicted in figure 1 .

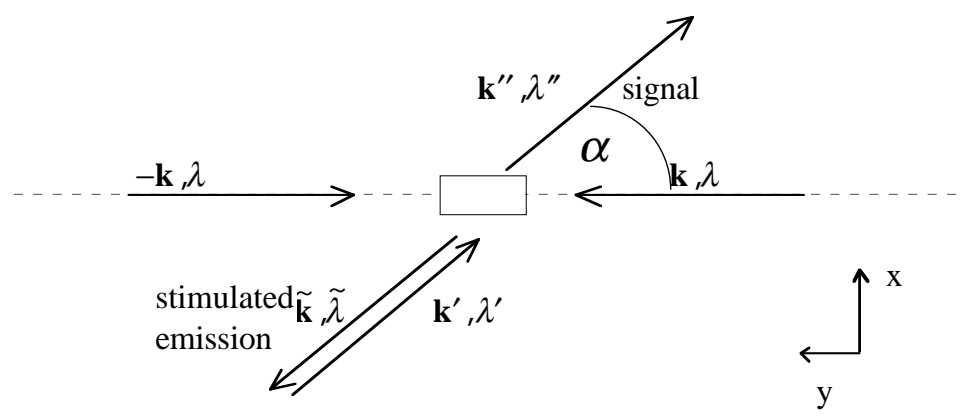

Figure 1. Beam geometry.

The five photons involved in each elementary interaction have the following characteristics. Two of the absorbed photons are of the same frequency $\omega$ and polarization $\lambda$, but propagate in opposite directions, $\hat{\boldsymbol{k}}$ and $-\hat{\boldsymbol{k}}$. The third absorbed photon has frequency $\omega^{\prime}$ and polarization $\lambda^{\prime}$, and its propagation direction $\hat{\boldsymbol{k}}^{\prime}$ forms an angle $\alpha$ with $-\hat{\boldsymbol{k}}$. The emitted photons have different characteristics. One is a stimulated photon of frequency $\omega$ and polarization $\tilde{\lambda}$, propagating in the $-\hat{\boldsymbol{k}}^{\prime}$ direction, while the signal photon is emitted with frequency $\omega^{\prime \prime}$, polarization $\lambda^{\prime \prime}$ and propagation direction $\hat{\boldsymbol{k}}^{\prime \prime} \equiv \hat{\boldsymbol{k}}^{\prime}$. Here, in common with phase conjugation experiments, the counterpropagating geometry for the $\hat{\boldsymbol{k}}$ and $-\hat{\boldsymbol{k}}$ beams offers an experimental opportunity for an intracavity implementation. In this case the general condition on the wavevectors is

$$
\omega^{\prime \prime} \hat{\boldsymbol{k}}^{\prime}-\omega \hat{\boldsymbol{k}}^{\prime}=\omega^{\prime} \hat{\boldsymbol{k}}^{\prime}+\omega \hat{\boldsymbol{k}}-\omega \hat{\boldsymbol{k}}
$$

with $\omega+\omega^{\prime}=\omega^{\prime \prime}$. The full set of photon parameters is summarized in table 1 .

Table 1. Photon characteristics.

\begin{tabular}{llc}
\hline & Frequency & Direction of propagation \\
\hline Absorbed photons & & \\
$(\boldsymbol{k}, \lambda)$ & $\omega$ & $\hat{\boldsymbol{k}}$ \\
$(-\boldsymbol{k}, \lambda)$ & $\omega$ & $-\hat{\boldsymbol{k}}$ \\
$\left(\boldsymbol{k}^{\prime}, \lambda^{\prime}\right)$ & $\omega^{\prime}$ & $\hat{\boldsymbol{k}}^{\prime}$ \\
Emitted photons & & \\
$(\tilde{\boldsymbol{k}}, \tilde{\lambda})$ & $\omega$ & $-\hat{\boldsymbol{k}}^{\prime}$ \\
$\left(\boldsymbol{k}^{\prime \prime}, \lambda^{\prime \prime}\right)$ & $\omega^{\prime \prime}$ & $\hat{\boldsymbol{k}}^{\prime}$ \\
\hline
\end{tabular}




\section{Quantum electrodynamical formulation}

The system formed by the molecules and the electromagnetic (EM) field, has an associated Hamiltonian $H$ expressible as

$$
H=H_{0}+V .
$$

The first term, whose eigenstates form the basis for the perturbation theoretical treatment, is

$$
H_{0}=\sum_{\xi} H_{\mathrm{mol}}^{\xi}+H_{\mathrm{rad}}
$$

where $H_{\text {mol }}^{\xi}$ is the Hamiltonian of molecule $\xi$ and $H_{\text {rad }}$ is the Hamiltonian of the field. The second term of (3.1) is the coupling

$$
V=\sum_{\xi} H_{\text {int }}^{\xi}
$$

between the molecules and the EM field. In the Power-Zienau-Woolley multipolar formulation, each molecule interacts separately with the field, intermolecular interaction being mediated by virtual photon coupling.

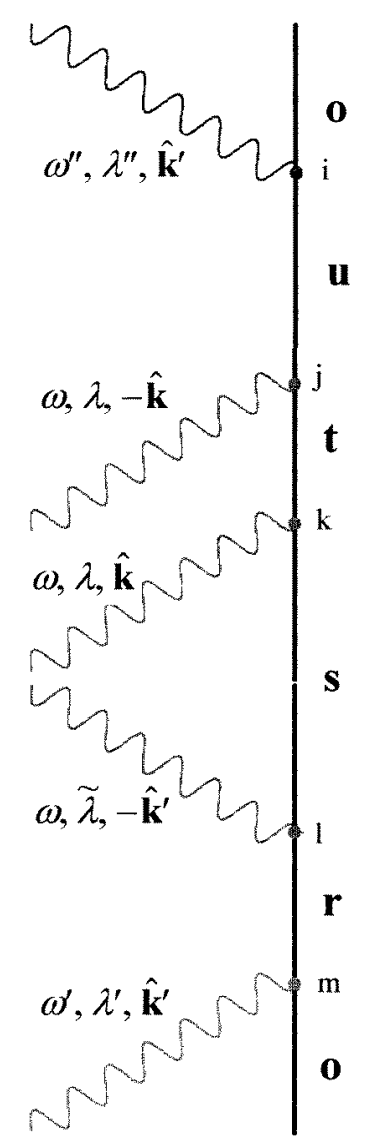

Figure 2. Feynman diagram representing the form of the interaction between the field and the molecules. 
To help develop in a detailed form the interaction between the field and the molecules, we introduce Feynman diagrams. Figure 2 represents one of the 120 different time-ordering graphs that contribute. With the aid of such graphs we can calculate the probability amplitude associated with transition between initial and final states specified as follows:

$|i\rangle=\left|E_{0} ; n_{+}(\boldsymbol{k}, \lambda) ; n_{-}(-\boldsymbol{k}, \lambda) ; n^{\prime}\left(\boldsymbol{k}^{\prime}, \lambda^{\prime}\right) ; \tilde{n}(\tilde{\boldsymbol{k}}, \tilde{\lambda})\right\rangle$

$|f\rangle=\left|E_{0} ;\left(n_{+}-1\right)(\boldsymbol{k}, \lambda) ;\left(n_{-}-1\right)(-\boldsymbol{k}, \lambda) ;\left(n^{\prime}-1\right)\left(\boldsymbol{k}^{\prime}, \lambda^{\prime}\right) ;(\tilde{n}+1)(\tilde{\boldsymbol{k}}, \tilde{\lambda}) ; 1\left(\boldsymbol{k}^{\prime \prime}, \lambda^{\prime \prime}\right)\right\rangle$

properly differentiating photons that have the same frequency but opposite directions of propagation. To calculate the transition rate $\Gamma$, through the application of the Fermi golden rule

$$
\Gamma=\left(\frac{2 \pi}{\hbar}\right)\left|M_{f i}\right|^{2} \rho
$$

our first task is to calculate the matrix element $M_{f i}^{\xi}$ for the molecule $\xi$, in terms of which the system matrix element $M_{f i}$ for $N$ molecules is given by

$$
M_{f i}=\sum_{\xi}^{N} M_{f i}^{\xi}
$$

For a five-wave mixing process the principal contribution to the matrix element is fifth order in the interaction term, i.e.

$$
\begin{aligned}
M_{f i}^{\xi} & =\left\langle f\left|U_{I}\left(t, t_{0}\right)\right| i\right\rangle_{\xi} \\
& \approx\left\langle f\left|\left\{\left(\frac{1}{\mathrm{i} \hbar}\right)^{5} \int_{t_{0}}^{t} \mathrm{~d} t_{1} \int_{t_{0}}^{t_{1}} \mathrm{~d} t_{2} \int_{t_{0}}^{t_{2}} \mathrm{~d} t_{3} \int_{t_{0}}^{t_{3}} \mathrm{~d} t_{4} \int_{t_{0}}^{t_{4}} \mathrm{~d} t_{5} \tilde{V}\left(t_{1}\right) \tilde{V}\left(t_{2}\right) \tilde{V}\left(t_{3}\right) \tilde{V}\left(t_{4}\right) \tilde{V}\left(t_{5}\right)\right\}\right| i\right\rangle_{\xi} \\
\tilde{V}\left(t_{i}\right) & =\exp \left[\mathrm{i} \frac{H_{0}\left(t_{i}-t_{0}\right)}{\hbar}\right] V \exp \left[-\mathrm{i} \frac{H_{0}\left(t_{i}-t_{0}\right)}{\hbar}\right]
\end{aligned}
$$

Calculating the five time integrals for the process described in section 2, and under the electric dipole approximation, we obtain

$$
\begin{aligned}
M_{f i}^{\xi}=(-\mathrm{i})( & \left.\frac{\hbar c}{2 \varepsilon_{0} V}\right)^{5 / 2}\left(k^{3} k^{\prime} k^{\prime \prime}\right)^{1 / 2}\left(n_{+} n^{\prime} n_{-}(\tilde{n}+1)\right)^{1 / 2} \\
& \times \bar{e}_{i}^{\prime \prime} \overline{\tilde{e}}_{j} e_{k}^{\prime} e_{l} e_{m} \chi_{i j k l m}^{\xi}\left(\omega^{\prime \prime} ;-\omega, \omega, \omega, \omega^{\prime}\right) \exp \left[\mathrm{i}\left(\boldsymbol{k}^{\prime}-\tilde{\boldsymbol{k}}-\boldsymbol{k}^{\prime \prime}\right) \cdot \boldsymbol{R}_{\xi}\right] .
\end{aligned}
$$

Here $\boldsymbol{e}^{\prime \prime}, \tilde{\boldsymbol{e}}, \boldsymbol{e}^{\prime}, \boldsymbol{e}$ are the polarization vectors of the photons, $\boldsymbol{R}_{\xi}$ is the position vector of the molecule $\xi$ and $\chi_{i j k l m}^{\xi}$ is its fifth-rank response tensor, whose explicit form is discussed in the following section.

From expression (3.6) and imposing wavevector matching, the total matrix element can finally be written as

$M_{f i}=(-\mathrm{i})\left(\frac{\hbar c}{2 \varepsilon_{0} V}\right)^{5 / 2}\left(k^{3} k^{\prime} k^{\prime \prime}\right)^{1 / 2}\left(n_{+} n^{\prime} n_{-}(\tilde{n}+1)\right)^{1 / 2} \bar{e}_{i}^{\prime \prime} \overline{\tilde{e}}_{j} e_{k}^{\prime} e_{l} e_{m} \sum_{\xi} \xi_{i j k l m}\left(\omega^{\prime \prime} ;-\omega, \omega, \omega, \omega^{\prime}\right)$.

Note that the effect of refractive dispersion will generally mean that exact collinearity of the $\boldsymbol{k}^{\prime}, \boldsymbol{k}^{\prime \prime}$ and $\tilde{\boldsymbol{k}}$ wavevectors cannot be achieved. However, fulfilment of wavevector matching under such conditions still generates a matrix element correctly represented by equation (3.10). 


\section{Structure of $\chi^{(4)}$}

Using the concise notation introduced recently by Naguleswaran and Stedman (1996), the nonlinear response tensor $\chi^{(4)}$ can be written compactly as follows:

$$
\chi_{\lambda_{1} \lambda_{2} \lambda_{3} \lambda_{4} \lambda_{5}}^{(4)} \equiv \chi_{f i}^{\pi\{\lambda, \eta \omega\}}=\sum_{\pi\{\lambda\}\{r\}} \frac{N_{f i}^{\pi\{\lambda\}\{r\}}}{D_{f i}^{\pi\{\eta \omega\}\{r\}}}
$$

where $\lambda=\left(\lambda_{1}, \lambda_{2}, \lambda_{3}, \lambda_{4}, \lambda_{5}\right)$ denotes the set of Cartesian coordinates in a molecular frame, $r=(r, s, t, u)$ the intermediate (virtual) states, with the linked permutation of each set of labels denoted by the braces $\pi\{\}$. Each numerator in equation (4.1) involves a product of transition electric dipole components:

$$
N_{f i}^{\pi\{\alpha\}\{s\}}=\mu_{\lambda_{1}}^{o u} \mu_{\lambda_{2}}^{u t} \mu_{\lambda_{3}}^{t s} \mu_{\lambda_{4}}^{s r} \mu_{\lambda_{5}}^{r o} .
$$

The 120 time orderings which contribute to the tensor are separable into two sets which differ by interchange of the fourth and fifth indices; as a consequence of the degeneracy in the corresponding beam frequencies, the tensor is rigorously index-symmetric in these indices (alone).

To be supported in a molecule of any given symmetry, components of $\chi^{(4)}$ must span the totally symmetric representation $\mathcal{D}^{(0-)}$ of the corresponding point group. Through its index symmetry property, the reducible representation of $\chi^{(4)}$ itself derives from a product of the representations of a third-rank odd-parity and a second-rank even-parity tensor,

$$
\begin{aligned}
\left(\mathcal{D}^{(0-)} \oplus 3 \mathcal{D}^{(1-)} \oplus 2 \mathcal{D}^{(2-)} \oplus \mathcal{D}^{(3-)}\right) \otimes\left(\mathcal{D}^{(0+)} \oplus \mathcal{D}^{(2+)}\right) \\
\quad=\left(3 \mathcal{D}^{(0-)} \oplus 9 \mathcal{D}^{(1-)} \oplus 9 \mathcal{D}^{(2-)} \oplus 7 \mathcal{D}^{(3-)} \oplus 3 \mathcal{D}^{(4-)} \oplus \mathcal{D}^{(5-)}\right)
\end{aligned}
$$

by the rules of angular momentum coupling (Andrews 1989). The result (4.3) obviously includes all weights $0 \leqslant j \leqslant 5$, leading (if there were a complete absence of molecular symmetry) to 162 independent components. In general, more than one of the weights will correlate with the totally symmetric representation of the molecular point group and lead to non-zero components for $\chi^{(4)}$; using methods well described by Barron (1982), these components and the relations between them are readily identified for any chosen symmetry. One immediate and general conclusion, based on the negative parity of the representations in (4.3), is that components of $\chi^{(4)}$ can assume finite values only where there is no molecular centre of symmetry. For this reason the process is forbidden in centrosymmetric molecules, and, for that matter, in centric crystals. In fluids, additional constraints become apparent once account is taken of bulk rotational symmetry (as we shall show in the following section) since full rotational symmetry supports $\mathcal{D}^{(0-)}$ alone.

The denominators in equation (4.1) involve differences between molecular state energies, $E_{r 0}=E_{r}-E_{0}$, and also the energies of the emitted and absorbed photons,

$D_{f i}^{\pi\{\eta \omega\}\{r\}}=\left(E_{u 0}-E_{4}-E_{3}-E_{2}-E_{1}\right)\left(E_{t 0}-E_{3}-E_{2}-E_{1}\right)\left(E_{s 0}-E_{2}-E_{1}\right)\left(E_{r 0}-E_{1}\right)$

where the energies $E_{i}(1 \leqslant i \leqslant 5)$ are

$$
E_{1}=\hbar \omega^{\prime} \quad-E_{2}=E_{3}=E_{4}=\hbar \omega \quad E_{5}=-\hbar \omega^{\prime \prime} .
$$

In frequency regions very substantially removed from single- or multi-photon absorption bands, approximate symmetry with respect to the permutations $\pi\{r\}$ would lead to Kleinman (complete) index symmetry in $\chi^{(4)}$. Then, its reducible representation collapses to $\left(\mathcal{D}^{(1-)} \oplus \mathcal{D}^{(3-)} \oplus \mathcal{D}^{(5-)}\right)$, with (at most) 21 independent components. 
Resonance is not a necessary condition for five-wave mixing to occur, though exploiting its onset through a judicious choice of frequency might expedite signal recovery, through reduction of one or more of the factors in (4.4). To avoid unphysical singularities and properly model the dispersion behaviour, it is appropriate to incorporate in each energy factor in (4.4) a term representing excited state damping. Then the difference in energy $E_{r} 0$ is replaced by

$$
\tilde{E}_{r 0}=E_{r 0}-\mathrm{i} \Gamma
$$

where $2 \pi / \Gamma_{r}$ is the lifetime of the molecular excited state $|r\rangle$, and the sign in equation (4.6), consistent with Cohen-Tannoudji et al (1992) properly accords with considerations of timereversal invariance (Andrews et al 1997). Under conditions of exact resonance, it would be appropriate to reformulate the interaction to include the effects of coherence decay.

\section{Distributional and rotational averaging}

In this section we impose two further conditions, both of which will be physically realized in the vast majority of experimental measurements. First we take account of the fact that the $N$ molecules in a fluid phase will be oriented randomly so that the ensemble response can be found by taking a rotational average, denoted below by angular brackets. Implementing this average on the modulus square of the matrix element gives a result which is expressible, through (3.6), as a sum of one-centre and two-centre terms:

$$
\begin{aligned}
\left\langle\left|\sum_{\xi}^{N} M_{f i}^{\xi}\right|^{2}\right\rangle & =\left\langle\sum_{\xi}^{N}\left|M_{f i}^{\xi}\right|^{2}\right\rangle+\left\langle\sum_{\xi^{\prime} \neq \xi}^{N} \sum_{f i}^{N} M_{f i}^{\xi} \bar{M}_{f i}^{\xi^{\prime}}\right\rangle \\
& =N\left\langle\left|M_{f i}^{\xi}\right|^{2}\right\rangle_{\xi}+N(N-1)\left\langle M_{f i}^{\xi}\right\rangle_{\xi}\left\langle\bar{M}_{f l}^{\xi^{\prime}}\right\rangle_{\xi^{\prime}} \\
& =N\left\langle\left|M_{f i}^{\xi}\right|^{2}\right\rangle+N(N-1)\left|\left\langle M_{f i}^{\xi}\right\rangle\right|^{2} .
\end{aligned}
$$

Here, in the last step, use is made of the fact that rotationally averaged parameters must be the same for all molecules of the ensemble. The first term in the result (5.1) corresponds to the incoherent addition of one-centre terms, given by the average squared modulus of the matrix element, while the second term represents the coherent interference of two-centre signals, for which rotational average is taken before the modulus squared. The persistence of the coherent term depends on fulfilling the condition of wavevector matching-failing this, the $\boldsymbol{R}_{\xi}$ position-dependent phase factor in (3.9) will carry through into the average to be conducted below, leading to effective cancellation of the two-centre terms. Under such conditions the incoherent (one-centre) response would certainly provide a signal, but one that is likely to be below the threshold of experimental detection. The assumption of phase matching thus allows neglect of the incoherent term, giving

$\left\langle\left|M_{f i}\right|^{2}\right\rangle \approx N^{2}\left|\left\langle M_{f i}^{\xi}\right\rangle\right|^{2}=N^{2}\left(\frac{\hbar c}{2 \varepsilon_{0} V}\right)^{5}\left(k^{3} k^{\prime} k^{\prime \prime}\right) n_{+} n^{\prime} n_{-}(\tilde{n}+1) \mid\left\langle\bar{e}_{\lambda_{1}}^{\prime \prime} \overline{\tilde{e}}_{\lambda_{2}} e_{\lambda_{3}}^{\prime} e_{\lambda_{4}} e_{\lambda_{5}}\left|\chi_{\lambda_{1} \lambda_{2} \lambda_{3} \lambda_{4} \lambda_{5}}\right|^{2}\right.$

from equation (3.10).

Implementation of the fifth-rank tensor rotational average is calculationally intricate, but readily achievable by methods based on isotropic tensor calculus. The method entails first referring all tensor components to a common molecule-fixed Cartesian frame (suitably denoted in (5.2) by the $\lambda$ indices) in which the susceptibility tensor components are invariant to rotation, then transforming the polarization components into a laboratory-fixed frame 
(denoted below by indices $i$ ) in which they too are invariant to molecular orientation. Thus we write

$$
\begin{aligned}
\left\langle\bar{e}_{\lambda_{1}}^{\prime \prime} \overline{\tilde{e}}_{\lambda_{2}} e_{\lambda_{3}}^{\prime} e_{\lambda_{4}} e_{\lambda_{5}}\right\rangle \chi_{\lambda_{1} \lambda_{2} \lambda_{3} \lambda_{4} \lambda_{5}} & =\left\langle\bar{e}_{i_{1}}^{\prime \prime} \overline{\tilde{e}}_{i_{2}} e_{i_{3}}^{\prime} e_{i_{4}} e_{i_{5}} \ell_{i_{1} \lambda_{1}} \ell_{i_{2} \lambda_{2}} \ell_{i_{3} \lambda_{3}} \ell_{i_{4} \lambda_{4}} \ell_{i_{5} \lambda_{5}}\right) \chi_{\lambda_{1} \lambda_{2} \lambda_{3} \lambda_{4} \lambda_{5}} \\
& =\bar{e}_{i_{1}}^{\prime \prime} \overline{\tilde{e}}_{i_{2}} e_{i_{3}}^{\prime} e_{i_{4}} e_{i_{5}} \chi_{\lambda_{1} \lambda_{2} \lambda_{3} \lambda_{4} \lambda_{5}}\left\langle\ell_{i_{1} \lambda_{1}} \ell_{i_{2} \lambda_{2}} \ell_{i_{3} \lambda_{3}} \ell_{i_{4} \lambda_{4}} \ell_{i_{5} \lambda_{5}}\right\rangle \\
& =\bar{e}_{i_{1}}^{\prime \prime} \overline{\tilde{e}}_{i_{2}} e_{i_{3}}^{\prime} e_{i_{4}} e_{i_{5}} \chi_{\lambda_{1} \lambda_{2} \lambda_{3} \lambda_{4} \lambda_{5}} I_{i_{1} i_{1} i_{2} i_{3} i_{4} i_{5} ; \lambda_{1} \lambda_{2} \lambda_{3} \lambda_{4} \lambda_{5}}^{(5)}
\end{aligned}
$$

in which the direction cosine $\ell_{i_{j} \lambda_{j}}$ is the $\left(i_{j}, \lambda_{j}\right)$ element of the Euler angle matrix relating the laboratory to the molecule-fixed frame. Expressed in this way, it is only these angles which vary with molecular orientation, so the problem reduces to finding the average of the product $\ell_{i_{1} \lambda_{1}} \ell_{i_{2} \lambda_{2}} \ell_{i_{3} \lambda_{3}} \ell_{i_{4} \lambda_{4}} \ell_{i_{5} \lambda_{5}}$, denoted by $I_{i_{1} i_{2} i_{3} i_{4} i_{5} ; \lambda_{1} \lambda_{2} \lambda_{3} \lambda_{4} \lambda_{5}}^{(5)}$. This is a linear combination of products of fifth-rank isotropic tensors referred to the two frames. Each isotropic tensor is the product of a Levi-Civita antisymmetric tensor and a Kronecker delta, and the coefficients of the linear combination can be taken directly from Andrews and Thirunamachandran (1977). The result is the following expression:

$$
\begin{aligned}
& \left\langle\left|M_{f i}\right|^{2}\right\rangle \approx\left(\frac{N}{30}\right)^{2}\left(\frac{\hbar c}{2 \varepsilon_{0} V}\right)^{2}\left(k^{3} k^{\prime} k^{\prime \prime}\right) n_{+} n^{\prime} n_{-}(\tilde{n}+1) \\
& \times \mid\left\{\left[\bar{e}^{\prime \prime} \cdot \overline{\tilde{e}} \times e^{\prime}\right](e \cdot e) \varepsilon_{\lambda_{1} \lambda_{2} \lambda_{3}} \delta_{\lambda_{4} \lambda_{5}}\right. \\
& +\left[\bar{e}^{\prime \prime} \cdot \overline{\tilde{e}} \times \boldsymbol{e}\right]\left(\boldsymbol{e}^{\prime} \cdot \boldsymbol{e}\right)\left(\varepsilon_{\lambda_{1} \lambda_{2} \lambda_{4}} \delta_{\lambda_{3}} \text { tambda } a_{5}+\varepsilon_{\lambda_{1} \lambda_{2} \lambda_{5}} \delta_{\lambda_{3} \lambda_{4}}\right) \\
& +\left[\bar{e}^{\prime \prime} \cdot e^{\prime} \times e\right](\overline{\tilde{e}} \cdot e)\left(\varepsilon_{\lambda_{1} \lambda_{3} \lambda_{5}} \delta_{\lambda_{2} \lambda_{4}}+\varepsilon_{\lambda_{1} \lambda_{3} \lambda_{4}} \delta_{\lambda_{2} \lambda_{5}}\right) \\
& \left.+\left[\overline{\tilde{e}} \cdot e^{\prime} \times e\right]\left(\bar{e}^{\prime \prime} \cdot e\right)\left(\varepsilon_{\lambda_{2} \lambda_{3} \lambda_{4}} \delta_{\lambda_{1} \lambda_{5}}+\varepsilon_{\lambda_{2} \lambda_{3} \lambda_{5}} \delta_{\lambda_{1} \lambda_{4}}\right)\right\}\left.\chi_{\lambda_{1} \lambda_{2} \lambda_{3} \lambda_{4} \lambda_{5}}\right|^{2} .
\end{aligned}
$$

In each term in equation (5.4) the contraction of the Levi-Civita and delta tensor product with the nonlinear susceptibility tensor generates a pseudoscalar $\left(\mathcal{D}^{(0-)}\right)$, i.e. a scalar of negative parity. It may be observed that, because the symmetry of the full rotation group now applies, contributions associated with any other weight disappear. Since pseudoscalars are quantities supported only by chiral species, i.e. molecules which possess neither a centre of symmetry nor any mirror plane or improper rotation axis, the process of interest is thus subject to more stringent symmetry constraints in fluids than in crystals. Note that in frequency regions where full Kleinman symmetry holds, these pseudoscalars necessarily vanish (because weight zero is then not represented in $\chi^{(4)}$ ) and the process becomes forbidden. In the next section the process will be analysed with regard to its dependence on beam polarization.

\section{Polarization behaviour}

\subsection{Linear polarization}

First we consider the case where all beams have linear polarization. The simplest geometry which leads to a finite signal is one in which photons derived from the counterpropagating beams $(\boldsymbol{k}$ and $-\boldsymbol{k})$ are polarized in the beam plane and the $\boldsymbol{k}^{\prime}$ beam is polarized perpendicularly to the plane. Allowing arbitrary linear polarizations for the other photons, we thus have

$e=\hat{\boldsymbol{e}}_{x} \quad \boldsymbol{e}^{\prime}=\hat{\boldsymbol{e}}_{z} \quad \boldsymbol{e}^{\prime \prime}=a \hat{\boldsymbol{e}}_{z}+b \hat{\boldsymbol{e}}_{\|} \quad e=c \hat{\boldsymbol{e}}_{z}+d \hat{\boldsymbol{e}}_{\|}$

where the directions $\hat{\boldsymbol{x}}$ and $\hat{\boldsymbol{z}}$ (right-hand rule) are defined in figure 1, $\hat{\boldsymbol{e}}_{\|}$necessarily satisfies

$$
\hat{k}^{\prime} \times \hat{e}_{z}=\hat{e}_{\|}
$$


and the real constants $a, b, c$ and $d$ are subject to the conditions

$$
\sqrt{a^{2}+b^{2}}=1 \quad \sqrt{c^{2}+d^{2}}=1 .
$$

Substituting in equation (5.4) we obtain

$$
\begin{aligned}
&\left\langle\left|M_{f i}\right|^{2}\right\rangle \approx\left(\frac{N}{30}\right)^{2}\left(\frac{\hbar c}{2 \varepsilon_{0} V}\right)^{5}\left(k^{3} k^{\prime} k^{\prime \prime}\right) n_{+} n^{\prime} n_{-}(\tilde{n}+1) \\
& \quad \times \mid \frac{1}{2} b d \sin (2 \alpha)\left(\varepsilon_{\lambda_{1} \lambda_{3} \lambda_{5}} \delta_{\lambda_{2} \lambda_{4}}+\varepsilon_{\lambda_{1} \lambda_{3} \lambda_{4}} \delta_{\lambda_{2} \lambda_{5}}\right. \\
&+\left.\varepsilon_{\lambda_{2} \lambda_{3} \lambda_{4}} \delta_{\lambda_{1} \lambda_{5}}+\varepsilon_{\lambda_{2} \lambda_{3} \lambda_{5}} \delta_{\lambda_{1} \lambda_{4}}\right)\left.\chi_{\lambda_{1} \lambda_{2} \lambda_{3} \lambda_{4} \lambda_{5}}\right|^{2}
\end{aligned}
$$

with the angle $\alpha$ as shown in figure 1 .

For conciseness we can define the following pseudoscalar coefficients:

$$
\begin{aligned}
& \varepsilon_{\lambda_{1} \lambda_{2} \lambda_{3}} \delta_{\lambda_{4} \lambda_{5}} \chi_{\lambda_{1} \lambda_{2} \lambda_{3} \lambda_{4} \lambda_{5}}=\chi_{1}^{(4)} \\
& \varepsilon_{\lambda_{1} \lambda_{2} \lambda_{4}} \delta_{\lambda_{3} \lambda_{5}} \chi_{\lambda_{1} \lambda_{2} \lambda_{3} \lambda_{4} \lambda_{5}}=\chi_{2}^{(4)} \\
& \varepsilon_{\lambda_{1} \lambda_{2} \lambda_{5}} \delta_{\lambda_{3} \lambda_{4}} \chi_{\lambda_{1} \lambda_{2} \lambda_{3} \lambda_{4} \lambda_{5}}=\chi_{3}^{(4)} \\
& \varepsilon_{\lambda_{1} \lambda_{3} \lambda_{4}} \delta_{\lambda_{2} \lambda_{5}} \chi_{\lambda_{1} \lambda_{2} \lambda_{3} \lambda_{4} \lambda_{5}}=\chi_{4}^{(4)} \\
& \varepsilon_{\lambda_{1} \lambda_{3} \lambda_{5}} \delta_{\lambda_{2} \lambda_{4}} \chi_{\lambda_{1} \lambda_{2} \lambda_{3} \lambda_{4} \lambda_{5}}=\chi_{5}^{(4)}
\end{aligned}
$$

and, noting the following which result from fundamental relationships between fifth-rank isotropic tensors (Andrews and Thirunamachandran 1977):

$$
\begin{aligned}
& \varepsilon_{\lambda_{2} \lambda_{3} \lambda_{4}} \delta_{\lambda_{1} \lambda_{5}} \chi_{\lambda_{1} \lambda_{2} \lambda_{3} \lambda_{4} \lambda_{5}}=\chi_{1}^{(4)}-\chi_{2}^{(4)}+\chi_{4}^{(4)} \\
& \varepsilon_{\lambda_{2} \lambda_{3} \lambda_{5}} \delta_{\lambda_{1} \lambda_{4}} \chi_{\lambda_{1} \lambda_{2} \lambda_{3} \lambda_{4} \lambda_{5}}=\chi_{1}^{(4)}-\chi_{3}^{(4)}+\chi_{5}^{(4)}
\end{aligned}
$$

we can finally express the result as

$$
\begin{aligned}
\left\langle\left|M_{f i}\right|^{2}\right\rangle=\left(\frac{N}{30}\right)^{2}\left(\frac{\hbar c}{2 \varepsilon_{0} V}\right)^{5}\left(k^{3} k^{\prime} k^{\prime \prime}\right) n_{+} n^{\prime} n_{-}(\tilde{n}+1) & \\
& \times\left|\frac{1}{2} b d \sin (2 \alpha)\left\{2\left(\chi_{1}^{(4)}+\chi_{4}^{(4)}+\chi_{5}^{(4)}\right)-\left(\chi_{2}^{(4)}+\chi_{3}^{(4)}\right)\right\}\right|^{2} .
\end{aligned}
$$

The result necessarily vanishes for $\alpha=0$, and suggests an angle of $\pm 45^{\circ}$ for the optimum signal.

\subsection{Circular polarization}

This case is not as simple as the former, though the calculational procedure is the same. Here we consider the following beam polarizations:

$$
\begin{array}{ll}
\boldsymbol{e}=\frac{1}{\sqrt{2}}\left(\hat{\boldsymbol{e}}_{x}+\mathrm{i} \hat{\boldsymbol{e}}_{z}\right) & \boldsymbol{e}^{\prime}=\frac{1}{\sqrt{2}}\left(\hat{\boldsymbol{e}}_{x}-\mathrm{i} \hat{\boldsymbol{e}}_{z}\right) \\
\boldsymbol{e}^{\prime \prime}=a \hat{\boldsymbol{e}}_{z}+b \hat{\boldsymbol{e}}_{\|} & \tilde{\boldsymbol{e}}=c \hat{\boldsymbol{e}}_{z}+d \hat{\boldsymbol{e}}_{\|} .
\end{array}
$$

Although the constants $a, b, c$ and $d$ still satisfy the normalization relation (6.3), they are now complex quantities. Here it is important to realize that the $k$ and $-\boldsymbol{k}$ beams have polarizations of opposite circularity, although they are described by the same polarization vector. In this case the final result is, as might be anticipated, more complicated then when 
working with linear polarization;

$$
\begin{aligned}
\left\langle\left|M_{f i}\right|^{2}\right\rangle=\left(\frac{N}{30}\right)^{2}\left(\frac{\hbar c}{2 \varepsilon_{0} V}\right)^{5}\left(k^{3} k^{\prime} k^{\prime \prime}\right) n_{+} n^{\prime} n_{-}(\tilde{n}+1) \\
\quad \times \mid\left\{(a d-b c)^{*} \frac{\sin \alpha}{\sqrt{2}}\left(\chi_{2}^{(4)}+\chi_{3}^{(4)}\right)+\left(-\frac{\mathrm{i} b^{*}}{\sqrt{2}}\right) \sin \alpha(d \cos \alpha+\mathrm{i} c)^{*}\left(\chi_{4}^{(4)}+\chi_{5}^{(4)}\right)\right. \\
\left.+\left(-\frac{\mathrm{i} d^{*}}{\sqrt{2}}\right) \sin \alpha(b \cos \alpha+\mathrm{i} a)^{*}\left(2 \chi_{1}^{(4)}-\chi_{2}^{(4)}-\chi_{3}^{(4)}+\chi_{4}^{(4)}+\chi_{5}^{(4)}\right)\right\}\left.\right|^{2}
\end{aligned}
$$

Notice that the linear combination of $\chi_{n}^{(4)}$ appearing in (6.9) differs from that in (6.7). In principle, measurements of the effect under both linear and circular polarization conditions thus offers additional information on the fourth-order susceptibility parameters.

\section{Discussion}

For the study of molecular fluids, the process described here offers a number of advantages over more familiar nonlinear optical processes, even in its simplest sumfrequency implementation. In particular, the greater flexibility in achieving wavevector matching affords considerable scope for study of the dependence on beam polarization and geometry. The angular dependence itself offers a ready means for characterizing the fivewave interaction, especially in the case of linear polarization. Together with the extensive possibilities for frequency tuning the process lends itself to a very complete characterization of fourth-order optical nonlinearity. Although we have focused on addressing the theory to isotropic molecular fluids, results for the substantially simpler case of an acentric crystalline material follow directly from the equations of section 3 .

To assess the likely magnitude of the signal, we can assume that the magnitudes of the transition dipole moment and energy denominator factors in equation (4.1) largely mirror those of the third-order susceptibility associated with four-wave mixing processes. It is then a straightforward matter to show that, compared with such processes, the five-wave interaction should generate a signal which is smaller by a factor typically of the order of $\gamma=I / J$. Here $I$ is the input irradiance and $J$ is a critical irradiance associated with departure from perturbation theory, i.e. the irradiance providing electric fields comparable to intramolecular Coulombic values, often quoted as around $10^{11} \mathrm{~W} \mathrm{~cm}^{-2}$. Thus, if studied with input pulses having a peak irradiance of $10^{10} \mathrm{~W} \mathrm{~cm}{ }^{-2}$, the five-wave process should offer output intensities around one tenth of those normally associated with phase conjugation and allied phenomena. We have shown that in isotropic fluids the five-wave interaction is forbidden in regions of transparency where Kleinman symmetry applies, although neither resonance nor pre-resonance is necessary for observation of the process, the corresponding signal enhancement will prove more than usually significant for optimizing signal detection.

\section{Acknowledgments}

We are pleased to acknowledge helpful discussions with Dr P Allcock. One of us (LDR) also gratefully acknowledges the award of a Research Studentship by the University of East Anglia (UEA). The femtosecond spectroscopy programme at UEA, of which this work is part, is funded by a grant from the Engineering and Physical Sciences Research Council. 


\section{References}

Allcock P and Andrews D L 1997 J. Phys. B: At. Mol. Opt. Phys. 30 3731-42

Andrews D L 1989 Spectrochim. Acta A 46 871-85

-1994 Nonlinear Opt. 8 25-32

Andrews D L and Hands I 1996 Chem. Phys. 23 277-94

Andrews D L, Naguleswaran S and Stedman G E 1997 work in progress

Andrews D L and Thirunamachandran T 1977 J. Chem. Phys. 67 5026-33

Barron L D 1982 Molecular Light Scattering and Optical Activity (Cambridge: Cambridge University Press)

Cohen-Tannoudji C, Dupont-Roc J and Grynberg G 1992 Atom-Photon Interactions (New York: Wiley)

Dubrovskii A V, Koroteev N I and Shkurinov A P 1992 JETP-Lett. 56 551-6

Koroteev N I 1995 Biospectroscopy $1341-50$

Naguleswaran S and Stedman G E 1996 J. Phys. B: At. Mol. Opt. Phys. 29 4027-40

Rentzepis P M, Giordmaine J A and Wecht K W 1966 Phys. Rev. Lett. 16 792-4

Shkurinov A P, Dubrovskii A V and Koroteev N I 1992 Phys. Rev. Lett. 70 1085-8 